\title{
Role of GILZ in the Kidney and the Cardiovascular System: Relevance to Cardiorenal Complications of COVID-19
}

\author{
Mahmood S. Mozaffari \\ Department of Oral Biology and Diagnostic Sciences, the Dental College of Georgia, Augusta University, Augusta, Georgia \\ Received July 24, 2020; accepted September 16, 2020
}

\begin{abstract}
Glucocorticoids are extensively used for a variety of conditions, including those associated with dysregulation of immune and inflammatory responses as primary etiopathogenic factors. Indeed, the proinflammatory cytokine storm of coronavirus disease 2019 (COVID-19) is the latest condition for which the use of a glucocorticoid has been advocated. Recognition of serious adverse effects of glucocorticoids has led to research aimed at unraveling molecular basis by which they impact immune and inflammatory events with the ultimate objective of devising novel therapies to circumvent glucocorticoids-related adverse outcomes. Consequently, glucocorticoid-induced leucine zipper (GILZ) protein was discovered and is increasingly recognized as the pivotal regulator of the effects of glucocorticoids on immune and inflammatory responses. Importantly, the advent of GILZ-based options raises the prospect of their eventual therapeutic use for a variety of conditions accompanied with dysregulation of immune and inflammatory responses and
\end{abstract}

associated target organ complications. Thus, the objective of this minireview is to describe our current understanding of the role of GILZ in the cardiovascular system and the kidney along with outcome of GILZ-based interventions on associated disorders. This information is also of relevance for emerging complications of COVID-19.

\section{SIGNIFICANCE STATEMENT}

Glucocorticoid-induced leucine zipper (GILZ) was initially discovered as the pivotal mediator of immune regulatory/suppressive effects of glucocorticoids. Since the use of glucocorticoids is associated with serious adverse effects, GILZ-based formulations could offer therapeutic advantages. Thus, this minireview will describe our current understanding of the role of GILZ in the kidney and the cardiovascular system, which is of relevance and significance for pathologies affecting them, including the multiorgan complications of coronavirus disease 2019.

\section{Introduction}

Endogenous glucocorticoids are well known for their diverse array of physiologic effects and regulation of body homeostasis, which are effects that are exerted through not only transcriptional regulation of genes but also epigenetic mechanisms (Bereshchenko et al., 2019). Recognition of the profound impact of glucocorticoids on immune and inflammatory mechanisms has led to their use in conditions caused by dysregulation of immune and inflammatory responses. However, glucocorticoids-based therapy causes a number of serious adverse outcomes, including elevation in plasma glucose and exacerbation of diabetes mellitus, increased blood pressure and worsening of cardiovascular pathologies (e.g., heart failure), osteoporosis, and steroid-induced psychosis among others; these adverse effects can lead to patient

\footnotetext{
This paper received no external funding.

https://doi.org/10.1124/jpet.120.000243.
}

noncompliance and discontinuation of therapy (O'Byrne and Mejza, 2018). The limitations of glucocorticoids therapy have led to a flurry of research to decipher molecular basis of their immune and inflammatory effects with the ultimate objective of harnessing their therapeutic efficacy (e.g., for sepsis) while avoiding their adverse effects (Vandewalle and Libert, 2020). This research has identified glucocorticoid-induced leucine zipper (GILZ) as a pivotal regulator of the impact of glucocorticoids on immune and inflammatory mechanisms, which are aspects that will be briefly described prior to description of the role of GILZ in the kidney and the cardiovascular system.

\section{Discovery of GILZ and Its Role in the Immune System}

Glucocorticoids and GILZ Induction. The seminal studies of D' Adamio et al. (1997) led to the initial discovery of GILZ as the product of a gene (TSC22d3) that is rapidly

\footnotetext{
ABBREVIATIONS: AKI, acute kidney injury; Bcl-xl, B-cell lymphoma-extra large; CD, cluster of differentiation; COVID-19, coronavirus disease 2019; DEXA, dexamethasone; ENaC, epithelial sodium channel; ERK, extracellular signal-regulated kinase; GILZ, glucocorticoidinduced leucine zipper; HUVEC, human umbilical vein endothelial cell; ICAM-1, intercellular adhesion molecule-1; IL, interleukin; ILC, innate lymphoid cell; LPS, lipopolysaccharide; $\psi_{\mathrm{m}}$, mitochondrial membrane potential; MAPK, mitogen-activated protein kinase; MCP-1, monocyte chemoattractant protein-1; MI, myocardial infarction; mpkCCD ${ }_{\mathrm{c} 14}$, mouse clonal cortical collecting duct principal cell line; NCC, sodium-chloride cotransporter; NF- $\kappa \mathrm{B}$, nuclear factor $\kappa$-light-chain enhancer of activated B cells; RMEC, retinal microvascular endothelial cell; SGK-1, serumglucocorticoid-regulated kinase 1; siRNA, small interfering RNA; TAT, transactivator of transcription; Th, T-helper lymphocyte; TNF, transforming growth factor; Treg, regulatory T lymphocyte; TSC, transforming growth factor $\beta$-stimulated clone box; TSC22D, transforming growth factor $\beta$-stimulated clone 22 domain.
} 
induced by DEXA. Accordingly, these investigators compared mRNA species that were expressed in DEXA-treated versus nontreated murine thymocytes and established GILZ as a new member of the leucine zipper family, which is highly expressed by normal lymphocytes (e.g., from thymus, lymph nodes, and spleen). They also showed that GILZ selectively protects $\mathrm{T}$ cells from apoptosis (induced by anti-CD3 monoclonal antibody) via inhibition of Fas and Fas-ligand expression. Shortly thereafter, a human homolog of murine GILZ was shown to be expressed by glucocorticoids in peripheral blood cells, including monocytes, granulocytes, and T cells; similarities of nucleotide sequence at $89 \%$ level in the entire mRNA and $97 \%$ in the coding region were shown for human versus mouse GILZ (Cannarile et al., 2001); several isoforms of GILZ have been reported for mouse and human, and their protein length is shown in Fig. 1 (Vétillard and Schlecht-Louf, 2018). Further investigation has shown that GILZ (about $15 \mathrm{kDa}$ ) and the isoform L-GILZ (about $28 \mathrm{kDa}$ ) are differentially expressed in a variety of cells, most notably hematopoietic cells (e.g., macrophages, lymphocytes) as well as epithelial cells, and that their expressions (particularly GILZ) are markedly upregulated by glucocorticoids (Bruscoli et al., 2010; Cari et al., 2015). Consistent with these observations, Ayyar et al. (2015) have shown baseline GILZ mRNA expression in a variety of rat tissues, including heart, kidney, lung, and adipose among others; indeed, adipose tissue displayed a robust circadian rhythm that was entrained with circadian oscillation of endogenous corticosterone. Furthermore, intramuscular injection of methylprednisolone $(50 \mathrm{mg} / \mathrm{kg})$ significantly increased GILZ mRNA expression in tested tissues (i.e., adipose, muscle, and liver). Assuming that the $50 \mathrm{mg} / \mathrm{kg}$
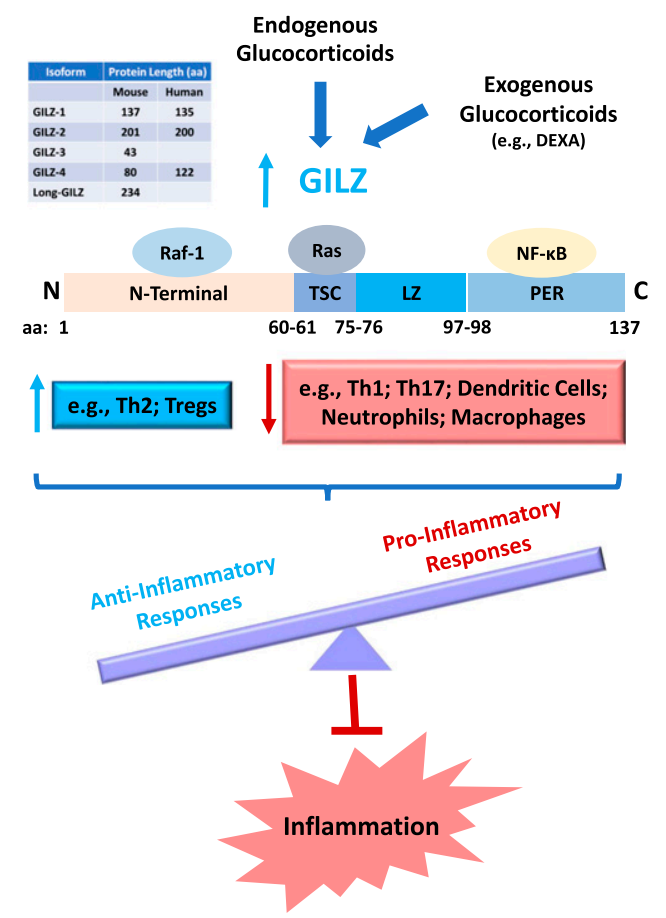

Fig. 1. Glucocorticoids markedly upregulate GILZ expression for which multiple isoforms are reported for both mouse and human as shown in the Table 1. In immune cells, GILZ interacts with several transcription factors, thereby determining their functional phenotypes. The outcome is believed to be primarily immune regulatory/suppressive effects, thereby reducing inflammation-aspects that are described in the text. represents the no-adverse-effect level of methylprednisolone for rat and based on guidelines for dose conversions (Nair and Jacob, 2016), about $8 \mathrm{mg} / \mathrm{kg}$ can be calculated for the human equivalent dose of methylprednisolone. Given biologic and physiologic differences among animal species versus humans and for safety considerations, it is recommended that the human equivalent dose be divided by a factor of 10 (i.e., $0.8 \mathrm{mg} / \mathrm{kg}$ for this scenario; Nair and Jacob, 2016). This dose is similar to the recommended (intramuscular) methylprednisolone dose of $40-60 \mathrm{mg}$ or about $0.6-0.9 \mathrm{mg} / \mathrm{kg}$ for human use, assuming an average body weight of $70 \mathrm{~kg}$. Thus, one would expect robust GILZ expression after methylprednisolone (or equivalent doses of other glucocorticoids) administration to human subjects. Nonetheless, although plasma GILZ mRNA level with respect to diurnal cortisol secretion for healthy human subjects has been determined (Yurtsever et al., 2019), it is imperative to establish GILZ expression levels in human subjects (e.g., in peripheral blood immune cells) in response to therapeutically used glucocorticoids. In consideration of this aspect, Ayyar et al. (2017) have described a quantitative model that integrates physiologic mechanisms (e.g., circadian rhythm and glucocorticoid tolerance) that ultimately determine tissue-specific regulation of corticosteroid-induced GILZ expression. Accordingly, authors showed that acute methylprednisolone injection significantly downregulated glucocorticoid receptor mRNA (40\%-80\%) but markedly increased GILZ mRNA expression (about 5-11 folds) in several rat tissues (e.g., muscle, lung, and adipose). In a more recent study, this group used GILZ expression as a biomarker to investigate sex-, tissue-, and estrous cycledependent variability in pharmacodynamics and pharmacokinetics of methylprednisolone (Ayyar et al., 2019).

Molecular Mechanisms of GILZ Actions. Studies focused on unraveling mechanisms of GILZ-induced effects have capitalized on information indicating that glucocorticoidsinduced inhibition of $\mathrm{NF}-\kappa \mathrm{B}$ transcriptional activity, in a variety of cells, is essential for inhibition of inflammatory responses; these include neutrophils, macrophages, monocytes, dendritic cells, and T lymphocytes. Initially, it was shown that GILZ inhibits NF- $\kappa$ B nuclear translocation and DNA binding, an effect related to a direct protein-to-protein interaction of GILZ with the p65 subunit of NF- $\kappa$ B. Furthermore, GILZ inhibited T-cell receptor-induced IL-2/IL-2 receptor expression. Subsequently, other mechanisms for GILZ-induced anti-inflammatory effects were proposed, such as interaction with other transcription factors, including c-Jun/c-Fos heterodimer (i.e., activator protein-1), Raf-1 proto-oncogene serine/threonine kinase, Ras protooncogene GTPase, and CCAAT-enhancer-binding proteins (Ayroldi et al., 2001; Vétillard and Schlecht-Louf, 2018; Bereshchenko et al., 2019). The current paradigm proposes that GILZ regulation of inflammation involves interaction of factors for which binding domains to GILZ are characterized (e.g., Raf-1 proto-oncogene serine/threonine kinase with N-terminal domain; Ras proto-oncogene GTPase with TSC domain; NF- $\kappa \mathrm{B}$ with proline and glutamic acid-rich domain; Fig. 1) as well as GILZ interactors with uncharacterized binding domains (e.g., c-Jun/c-Fos, small mothers against decapentaplegics, CCAAT-enhancer-binding proteins) (Bereshchenko et al., 2019).

GILZ-Induced Regulation of Immune Cells. At the cellular level, although GILZ increases Th-2 response and 
upregulates development of Tregs, it exerts inhibitory effects on other immune cells, including macrophages, neutrophils, dendritic cells, Th-1, Th-17, and B lymphocytes (Bereshchenko et al., 2019; Fig. 1); this minireview will suffice to describe the roles of GILZ in macrophages and T lymphocytes as examples of cells of innate and adaptive immunity, respectively.

With respect to macrophages, it is shown that toll-like receptor activation of human macrophages via myeloid differentiation primary response 88-and tristetraprolin-dependent mechanisms downregulates GILZ likely via mRNA destabilization (Hoppstädter et al., 2012). Utilizing a myeloid-specific GILZ knockout mouse model thereafter, it was shown that GILZ-deficient macrophages exhibit an exaggerated ERKdependent increase in LPS responsiveness, as revealed by increased expression of transforming growth factor (TNF)- $\alpha$ and IL-1 $\beta$. Furthermore, the LPS-induced activation of macrophages was reduced upon their pretreatment with low-dose LPS, which is a phenomenon referred to as endotoxin tolerance (Hoppstädter et al., 2015). Interestingly, although GILZ mRNA was stabilized in LPS-tolerant macrophages, ERK activation was markedly reduced. On the other hand, GILZ knockout macrophages displayed significant reduction in desensitization. Furthermore, systemic low-dose LPS pretreatment of GILZ knockout mice reduced proinflammatory mediator expression in both serum and tissues upon high-dose LPS treatment; however, high-dose LPS-induced cytokine expression was preserved in tolerized GILZ knockout mice. Based on their collective observations, authors concluded that GILZ is a pivotal regulator of macrophage function (Hoppstädter et al., 2015). The observation that TSC22D3 mRNA expression is downregulated in white blood cells of patients with sepsis prompted Ballegeer et al. (2019) to explore the role of GILZ using the transgenic mice overexpressing GILZ in the cecal ligation and puncture model of sepsis. Accordingly, they showed improved survival in those animals, which was attributed to reduced bacterial counts in the blood and increased TSC22D3 mRNA expression in peritoneal exudate cells. Furthermore, they showed increased phagocytosis of CD45 + GILZ transgenic cells compared with CD45 + GILZ wild-type cells; among other cells, macrophages express CD45. A more recent study showed that 1) monocytes and macrophages from patients and mice with septic shock have significantly reduced GILZ expression than those of their controls; 2) septic transgenic mice with increased GILZ expression restricted to monocytes and macrophages displayed lower frequencies of inflammatory monocytes than their littermates and lower plasma levels of inflammatory cytokines, which are effects that were accompanied with lower blood bacterial counts; 3) upregulation of GILZ in monocytes and macrophages increased their phagocytic capacity; and 4) septic mice with increased GILZ expression in their monocytes and macrophages had increased survival rates (Ellouze et al., 2020). Based on their collective findings, the authors propose an essential role for GILZ and monocytes/macrophages in pathogenesis of septic shock. Indeed, given the limitations of glucocorticoids (e.g., adverse effects and resistance), it is suggested that GILZ-based options (e.g., cell-permeable GILZ, synthetic GILZ-derived peptide, CRISPR-based transcriptional activators) may represent viable treatment modalities for sepsis (Vandewalle and Libert, 2020). Studies exploring regulation of macrophage function indicate that GILZ downregulation via myeloid differentiation primary response 88-dependent and -independent mechanisms is a consequence of decreased mRNA, reduced translation, and/or protein stability, with effects promoting macrophage activation (Hoppstädter et al., 2019). This is seemingly consistent with the notion that GILZ promotes polarization of macrophages favoring M2-like functional phenotype (IL-4 differentiated), whereas GILZ knockout mice display higher M1-like markers associated with lower IL-10 levels compared with M1-differentiated cells from wild-type mice (Vago et al., 2020); M1 and M2 are considered to be proinflammatory and anti-inflammatory functional phenotypes of macrophages. Indeed, given the complexities of GILZ actions in macrophages, it is suggested that their targeting (e.g., via nanoparticle delivery systems) to achieve upregulation or downregulation of GILZ expression may offer novel therapeutic options for immunomodulation (Hoppstädter and Kiemer, 2015). Interestingly, given the pivotal role of GILZ in macrophage function, it has been postulated that lack of GILZ induces "macrophaging," which contributes to the chronic low-grade inflammation associated with aging (i.e., "inflammaging"). Accordingly, it is shown that macrophages from aged mice display functional phenotype resembling that of young GILZ knockout mice, thereby implicating dysregulation of GILZ in the aging process (Valbuena Perez et al., 2020).

With respect to T lymphocytes, glucocorticoid-induced GILZ expression is associated with multiple effects, including modulation of naïve T-cell differentiation, thereby promoting inhibition of Th-1 and Th-17 cell development while inducing Tregs and Th- 2 cells. Nonetheless, endogenous expression of GILZ in naïve $\mathrm{T}$ cells is suggestive of effects independent of glucocorticoids (Cannarile et al., 2019). Initial studies using GILZ-overexpressing hybridoma T-cell line 3DO indicated inhibition of CD3-mediated apoptosis (D'Adamio et al., 1997; Ayroldi et al., 2001). Thereafter, it was shown that IL-2 deprivation transiently increases GILZ expression in T lymphocytes, thereby conferring protection from the onset of apoptosis; this effect was dependent on GILZ-induced inhibition of forkhead box-O3 transcriptional activity, which in turn downregulated not only Bim expression but also GILZ itself, which is suggestive of an autoregulatory loop (AsselinLabat et al., 2004). Subsequent studies showed that GILZ expression in human dendritic cells (e.g., in response to IL-10 or glucocorticoids) restricts their maturation process and inhibits antigen-specific T-cell response (Cohen et al., 2006). More recently, in vivo studies showed that CD11c-GILZ ${ }^{\text {hi }}$ transgenic mice display an age-dependent accumulation of forkhead box-p3 ${ }^{+}$Tregs in the spleen (Calmette et al., 2014). As recently reviewed by Cannarile et al. (2019), cumulative evidence indicates that GILZ promotes a tolerogenic dendritic cell phenotype and inhibits antigen-presenting functions, thereby resulting in inhibition of T-cell activation but induction of Treg development. Furthermore, in naïve T cells, GILZ modulates their differentiation, thereby promoting induction of Th-2 and Tregs while inhibiting Th-1 cells and exerting context-specific inhibition or stimulation of Th-17 cell development. The translational outcome of immune suppressive/regulatory function of GILZ in T lymphocytes is exemplified by reports that its overexpression in these cells inhibits tissue damage and spinal cord injury (Esposito et al., 2012) and that GILZ regulates Th-17 responses to restrain IL-17-mediated 
skin inflammation-a finding of clinical relevance to psoriasis (Jones et al., 2015).

In summary, it is increasingly apparent that GILZ functions as a factor essential for the maintenance of quiescent immune cells in both the innate and adaptive arms of immunity, thereby promoting primarily immune regulatory/suppressive functional phenotypes.

\section{GILZ and the Kidney}

The demonstration that GILZ is expressed in the kidney led to investigation of its role in renal sodium and fluid handling. Using serial analysis of gene expression, Robert-Nicoud et al. (2001) showed GILZ as one of the candidate proteins mediating physiologic responses of a highly differentiated mouse clonal cortical collecting duct principal cell line $\left(\mathrm{mpkCCD}_{\mathrm{c} 14}\right)$ to aldosterone and vasopressin. More recently, using genomewide analysis of murine renal distal convoluted tubular cells, it was shown that both GILZ and SGK-1 were among the five direct target genes of mineralocorticoid receptor activation (Ueda et al., 2014). Given the prominent impact of aldosterone on kidney function via interaction with the mineralocorticoid and the glucocorticoid receptors, Muller et al. (2003) examined its effects on urinary electrolyte excretions in the context of assessment of expression levels of the following aldosteroneinduced transcripts: the $\alpha$ subunit of ENaC, SGK-1, and GILZ. Accordingly, they showed that increased SGK-1 expression (in the collecting ducts) is a primary event in aldosterone-induced renal responses (i.e., sodium reabsorption but potassium excretion) and that expression of $\alpha \mathrm{ENaC}$ and/or GILZ may play a permissive role in these responses. Subsequent studies more firmly established a role for GILZ in regulation of renal epithelial sodium transport as follows: 1) GILZ expression was rapidly increased in response to aldosterone in $\mathrm{mpkCCD}_{\mathrm{c} 14}$ cells, and in turn, GILZ markedly stimulated ENaC-mediated sodium transport via inhibition of ERK signaling; 2) GILZ transfection in mpkCCD $\mathrm{c}_{\mathrm{c} 14}$ cells inhibited phospho-ERK level and increased transepithelial sodium transport; 3) GILZ transfection in Xenopus oocytes with activated ERK inhibited phospho-ERK level and stimulated ENaC-mediated sodium current in a manner similar to that observed for pharmacological inhibition of ERK; and 4) GILZ expression markedly increased cell surface expression of $\mathrm{ENaC}$ in epidermal growth factor-treated mammalian kidney epithelial cells (human embryonic kidney-293) (Soundararajan et al., 2005). Others have suggested that GILZ and SGK-1 negatively regulate tonic inhibition of $\mathrm{ENaC}$ and that the disinhibition mechanism leads to dissemination of aldosterone effects to enhance renal sodium reabsorption (Bhalla et al., 2006). Nonetheless, assessment of TSC22D gene responses to hyperosmolality in kidney cells indicates that the TSC22D3 transcripts, including GILZ, do not play a role in adaptation of renal cells to hypertonicity (Fiol et al., 2007). Interestingly, sexual dimorphism has been reported with respect to expression of several mineralocorticoid target genes, including that for GILZ, during renal development with potential long-term consequences, such as blood pressure control. Indeed, renal GILZ expression was downregulated in kidneys of female mice (along with lower blood pressure), which was attributed to upregulation of renal metabolism and clearance of glucocorticoids (Dumeige et al., 2017).
The role of GILZ in kidney function has been more firmly established using the GILZ-deficient mice that showed subtle water and electrolyte abnormalities (Suarez et al., 2012). Utilizing the GILZ knockout mouse model, Rashmi et al. (2017) reported the following features compared with wildtype mice: 1) hyperkalemia and lower fractional excretion of potassium, 2) increased sensitivity to thiazide-induced inhibition of NCC, 3) no change in blood pressure despite increased NCC activity, 4) more robust adaptation to sodium deprivation without significant increase in plasma renin activity, and 5) hyperkalemia after prolonged sodium restriction. They also showed that treatment with GILZ inhibits NCC activity of human embryonic kidney-293T cells, in part via inhibition of SPS1-related proline/alanine-rich kinase phosphorylation. Based on their collective findings, authors concluded that 1) GILZ promotes potassium secretion by inhibiting NCC and increasing distal delivery to epithelial sodium channel and 2) GILZ knockout mice display features resembling familial hyperkalemic hypertension (Rashmi et al., 2017).

In pursuit of better understanding of molecular mechanisms of essential hypertension, a recent study investigated the role of GILZ in relation to perinatal epigenetic programming (Dumeige et al., 2020). Authors reported that prematurity increased renal mRNA levels for GILZ and $\alpha \mathrm{ENaC}$ without changes in either mineralocorticoid or glucocorticoid receptor expression. Interestingly, although high expression levels of GILZ and $\alpha \mathrm{ENaC}$ were not sustained in adulthood, former preterm male mice developed hypertension by about 6 months of age. Furthermore, offspring of the preterm group (F2 and F3) also displayed higher blood pressure, which was accompanied with increased renal GILZ mRNA, without changes in plasma corticosteroid levels or mineralocorticoid or glucocorticoid receptor expression. However, GILZ promoter DNA methylation was reduced with a negative correlation between methylation and expression. In light of these observations, further investigation of the role of GILZ in a myriad of long-term complications and pathologies attributed to prematurity is warranted.

The aforementioned studies explored the role of endogenous GILZ in the kidney. However, the advent of a cell-permeable GILZ fusion protein [i.e., transactivator of transcription (TAT)-GILZ; Delfino et al., 2004] has provided a unique opportunity to explore the impact of therapeutic GILZ in pathologic conditions. Indeed, the landmark study of Cannarile et al. (2009) was the first study to show in vivo efficacy of therapeutic GILZ delivery. Accordingly, authors showed that prophylactic TAT-GILZ fusion protein prevents the development of colitis induced by intracolonic administration of dinitrobenzene sulfonic acid; indeed, the treatment was associated with marked improvement of the wasting disease and significant reduction in inflammation based on macroscopic and histologic examinations of the colon as well as colonic myeloperoxidase activity. Interestingly, therapeutic GILZ delivery elicited effects that paralleled those of DEXAtreated animals, prompting authors to further show that DEXA treatment markedly upregulates GILZ expression in mucosal $\mathrm{T}$ lymphocytes and leading them to suggest that DEXA-induced anti-inflammatory effects in this model of colitis are partly related to increased GILZ expression. Importantly, authors also showed that TAT-GILZ fusion protein exerts protective effects against fully developed colitis 
in IL-10 knockout mice as assessed by histologic examination, colonic myeloperoxidase activity, $\mathrm{TNF}-\alpha$, and macrophage inflammatory protein-1 $\alpha$. Thereafter, Vago et al. (2015) reported that administration of TAT-GILZ fusion protein induces a robust proapoptotic program that resolves neutrophilic inflammation in a murine model of LPS-induced pleurisy.

Our recent investigation of the impact of GILZ delivery in AKI was prompted by the wealth of knowledge regarding the influence of GILZ on immune and inflammatory mechanisms as briefly described above and the fact that AKI, which is a condition associated with dysregulation of immune and inflammatory responses, remains a major clinical problem affecting more than $50 \%$ of patients who are critically ill with detrimental short- and long-term effects (Ronco et al., 2019). Thus, we initially explored the impact of GILZ delivery on polarization of T lymphocytes and neutrophils in the context of its potential renoprotective effect in AKI; although polarization of T lymphocytes to regulatory and effector subtypes is well established, neutrophil polarization to proinflammatory and anti-inflammatory subtypes (i.e., N1 and N2, respectively) is a more recent finding (Fridlender et al., 2009; Ma et al., 2016). Thus, using a murine model of AKI simulated by renal ischemia-reperfusion injury, we showed that AKI increased N1 and Th-17 cells but reduced N2, Tregs, and Treg17 cells (which counteract Th-17-specific responses) in association with increased $\mathrm{IL}-17^{+}$but reduced $\mathrm{IL}-10^{+}$cells accompanied with the disruption of $\psi_{\mathrm{m}}$ and increased apoptosis/necrosis (of whole kidney cell preparations) compared with sham kidneys. TAT-GILZ - as compared with TAT_treatment reduced N1 and Th-17 cells but restored N2 and Tregs without significantly affecting Treg17 cells, and this was associated with a reduction in $\mathrm{IL}-17^{+}$cells but an increase in $\mathrm{IL}-10^{+}$cells; TATGILZ also caused less disruption of $\psi_{\mathrm{m}}$ and reduced cell death in AKI. Importantly, TAT-GILZ increased perfusion of the ischemic-reperfused kidney but reduced tissue edema compared with TAT. Utilizing splenic T cells and bone marrowderived neutrophils, we further showed marked reduction in the proliferation of Th cells in response to TAT-GILZ compared with response to TAT. Collectively, the results indicate that GILZ exerts renoprotection accompanied by upregulation of the regulatory/suppressive arm of immunity in AKI likely via regulating crosstalk between $\mathrm{T}$ cells and neutrophils (Baban et al., 2018). More recently, we focused on the role of innate lymphoid cells (ILCs) and the impact of therapeutic GILZ in AKI. ILCs are largely tissue-resident archetypal cells of the immune system and recognized as important early sentinel cells at the interface of innate and adaptive immunity. They are composed of three major subtypes based on their transcription factor and cytokine profiles; although proinflammatory effects are attributed to ILC1s and ILC3s, ILC2s contribute to resolution of inflammation (Crome and Ohashi, 2018). We showed that ILC1s and ILC3s were increased, but ILC2s were decreased in the murine model of AKI; this pattern was reversed by TAT-GILZ compared with TAT treatment. Furthermore, GILZ-positive subsets of ILCs were reduced in AKI, which is an effect less marked for ILC2s (Baban et al., 2020). ILCs are believed to crosstalk with various immune cells and may exert effects on other cells, including epithelial cells and fibroblasts (Symowski and Voehringer 2017). Thus, the ability of GILZ to influence not only subtypes of ILCs but also those of T lymphocytes and neutrophils in AKI is suggestive of broader effects of GILZ on immune cells and potentially their interactions in regulation of response to injury in the kidney, which are aspects that remain to be established. Importantly, however, the impact of therapeutic GILZ on other pathologies of the kidney remains to be established; these include septic and drug-induced AKI, glomerulonephritis, and diabetic nephropathy among others.

In summary, endogenous GILZ is believed to regulate renal function primarily in relation to electrolyte homeostasis. Furthermore, the ability of GILZ to regulate/modulate immune and inflammatory response likely contributes to the cytoprotective and renoprotective effects of therapeutic GILZ in AKI (Table 1). Nonetheless, determination of the role of endogenous GILZ in conditions that markedly impact renal function (e.g., diabetes mellitus, hypertension) and the outcome of exogenous GILZ therapy on nephropathies of various causes represent fertile areas of investigation.

\section{GILZ and the Cardiovascular System}

Several studies have explored the role of GILZ in endothelial cells and in relation to modulation of immune and inflammatory responses. An earlier study focused on gene expression of HUVECs subjected to shear stress (McCormick et al., 2001). Accordingly, using microarray screening, 52 genes were identified with significantly altered expression profiles in response to shear stress compared with matched stationary controls. GILZ gene was among the 23 genes that displayed enhanced expression by more than 2-fold. Authors indicated that their observations are suggestive of potential mechanisms for enhanced nitric oxide production in endothelial cells. Nonetheless, a direct link between endothelial nitric oxide production and GILZ remains to be established; however, lymphocytes overexpressing GILZ are reported to have reduced inducible nitric oxide synthase activity and nitrotyrosine generation (Esposito et al., 2012). The role of GILZ in endothelial cells was more directly investigated by transient transfection in HUVECs (Cheng et al., 2013). Accordingly, it was shown that 1) GILZ overexpression in HUVECs significantly decreased TNF-stimulated leukocyte rolling, adhesion, and transmigration, and these effects were accompanied with reduced expression of E-selectin, ICAM-1, chemokine ligand 2, C-X-C motif chemokine ligand 8, and IL-6 - however, silencing of endogenous GILZ in glucocorticoid-treated HUVECs did affect their capacity to support leukocyte interactions; 2) GILZ overexpression in a human microvascular endothelial cell line inhibited TNF-inducible NF- $\kappa$ B activity; and 3) GILZ overexpression inhibited TNF-induced stimulation of p38, ERK, and c-Jun N-terminal kinase MAPKs and also increased expression of the MAPK phosphatase-1. Furthermore, exogenous GILZ (induced via transient transfection) inhibited TNFinduced NF- $\mathrm{B}$ p65 DNA binding without affecting p65 nuclear translocation suggestive of differences in actions of exogenous GILZ in endothelial cells than other cell types (e.g., $\mathrm{T}$ cells in which transfection-induced GILZ increase results in direct binding to p65 subunit, thereby preventing nuclear translocation and subsequent DNA binding). Based on their collective observations, authors suggested that induction of GILZ expression in endothelial cells may represent a novel therapeutic modality to curb inflammatory leukocyte recruitment (Cheng et al., 2013). Of particular relevance to leukocyte adhesion cascade is the demonstration that expressions of 
TABLE 1

Some actions of GILZ in the kidney and the cardiovascular system along with the outcome of GILZ delivery on related pathologies; these aspects are detailed in the text

\begin{tabular}{|c|c|c|}
\hline Heart & Kidney & Vasculature \\
\hline $\begin{array}{l}\text { - Cytoprotection in a cardiomyocyte injury model: } \\
\text { enhancement with GILZ overexpression, and abrogation } \\
\text { with GILZ knockdown } \\
\text { - Therapeutic GILZ in the murine model of MI: } \\
>\text { Increased Tregs but reduced Th-17 cells } \\
>\text { Preservation of } \psi_{\mathrm{m}} \text { in whole cardiac cell preparations } \\
>\text { Prevention of apoptotic and necrotic cell death in whole } \\
\text { heart cell preparations }\end{array}$ & $\begin{array}{l}\text { - Modulation of ENaC expression } \\
\text { - Modulation of electrolyte } \\
\text { homeostasis } \\
\text { - Sexual dimorphism and GILZ } \\
\text { expression } \\
\text { - Perinatal epigenetic programming } \\
\text { - Therapeutic GILZ in AKI: } \\
>\text { Promotion of regulatory } \\
\text { neutrophils and Tregs } \\
>\text { Restoration of ILC2s but reduction } \\
\text { of ILC1s and ILC3s } \\
>\text { Preservation of } \psi_{\mathrm{m}} \text { and reduced cell } \\
\text { death in whole kidney cell } \\
\text { preparations } \\
>\text { Improved renal perfusion and } \\
\text { reduced tissue edema }\end{array}$ & $\begin{array}{l}\text { - GILZ-induced modulation/regulation of } \\
\text { inflammatory cytokine expression in } \\
\text { HUVECs and RMECs } \\
\text { - GILZ-induced modulation/inhibition of } \\
\text { leukocyte adhesion and transmigration } \\
\text { - Modulation of GILZ expression by shear force } \\
\text { in endothelial cells } \\
\text { - GILZ-induced protection against sepsis }\end{array}$ \\
\hline
\end{tabular}

both GILZ and developmental endothelial locus-1, which is a pivotal negative regulator of leukocyte transmigration, are reduced in association with marked inflammatory cell infiltrates in salivary glands of a rat model of Sjögren syndrome (Qin et al., 2016); nonetheless, a pathogenic role for dysregulation of GILZ in developmental endothelial locus-1 expression and/or function remains to be established. Others have explored the role of GILZ in RMECs since inflammation and endothelial cell injury are prominent mechanisms in development of diseases of the retina, including ischemic retinal vasculopathy, posterior uveitis, and diabetic retinopathy $(\mathrm{Gu}$ et al., 2017). Accordingly, LPS-induced reduction in GILZ protein in RMECs has been shown in a time- and dosedependent fashion accompanied with upregulation of ICAM1 and MCP-1 expressions; these effects were prevented by GILZ overexpression, whereas GILZ silencing exacerbated them. Furthermore, GILZ overexpression prevented LPSinduced NF- $\kappa$ B p65 nuclear translocation in RMECs, which was mediated by enhanced p65 dephosphorylation, which was an effect that further downregulated ICAM-1 and MCP-1 expression (Gu et al., 2017). More recently, it was shown that intravitreal injection of a synthetic GILZ peptide (GILZ98134) inhibited ocular inflammation in a rat model of endotoxininduced uveitis as indexed by reduced expression of IL-1 $\beta$, TNF- $\alpha$, MCP-1, and ICAM-1 (Guo et al., 2020b). The clinical relevance and significance of anti-inflammatory effects of GILZ in endothelial cells were reported by Hahn et al. (2014), who demonstrated significant reductions in GILZ mRNA and protein expressions in degenerated aortocoronary saphenous vein bypass grafts, which also displayed increased MCP-1 and toll-like receptor-2 expression as indicators of inflammation compared with healthy veins. Authors also showed decreased GILZ expression in HUVECs (and macrophages) treated with TNF- $\alpha$ in a tristetraprolin- and p38 MAPKdependent manner. Reduction of GILZ expression via siRNA resulted in increased $\mathrm{NF}-\kappa \mathrm{B}$ activity and inflammatory gene expression. TNF- $\alpha$-activated HUVECs displayed induction of tristetraprolin similar to that of degenerated vein bypass grafts. Interestingly, laminar shear stress increased GILZ mRNA and protein expressions in HUVECs and also counteracted TNF- $\alpha$-induced tristetraprolin expression and GILZ downregulation. Furthermore, laminar shear stress upregulated MAPK phosphatase-1 that was downregulated in degenerated vein bypass grafts. Authors concluded that inflamed vasculature displays diminished tristetraprolindependent GILZ expression (Hahn et al., 2014). Collectively, these observations are suggestive of a pathogenic role for dysregulation of GILZ in cardiovascular disease.

With respect to the heart, DEXA-induced GILZ protein expression has been shown in a time- and dose-dependent fashion in primary cultured rat cardiomyocytes and H9c2(2-1) cell line; this study also showed increased GILZ protein expression in the myocardium of DEXA-treated mice (Aguilar et al., 2013). Thereafter, the same group reported cytoprotective effects of GILZ in doxorubicin-induced cardiac injury (Aguilar et al., 2014). Accordingly, authors showed corticosterone-induced GILZ expression in cardiomyocytes and that siRNA knockdown of GILZ abrogates corticosterone-induced reduction of apoptosis in response to doxorubicin. Furthermore, GILZ overexpression by transfection protects cardiomyocytes against doxorubicininduced apoptosis associated with prevention of mitochondrial release of cytochrome $c$ and caspase- 3 cleavage while increasing prosurvival protein Bcl-xl; however, siRNA targeting Bcl-xl prevented corticosterone-induced cytoprotection. Based on their collective findings, authors concluded that GILZ exerts cytoprotective effects (e.g., via Bcl-xl induction) in cardiomyocytes.

Utilizing the cryoinjury-induced murine model of MI, our group explored the status of GILZ and the outcome of intramyocardial delivery of GILZ-overexpressing mesenchymal stem cells in this condition; the cryoinjury model of MI is particularly suited for stem cell studies because intramyocardial injections can be accomplished in a well defined region of injury (Baban et al., 2017). Accordingly, we showed that MI markedly reduced cardiac GILZ expression accompanied with 1) increase in Th-17 cells but decrease in Tregs and 2) disruption of $\psi_{\mathrm{m}}$ associated with significant increases in apoptotic and necrotic cell death in whole cardiac cell preparations; these effects were markedly reversed by intramyocardial 
GILZ delivery. These observations are of clinical relevance and significance since MI predisposes to adverse cardiac remodeling and long-term sequels, such as heart failure, and interventions early post-MI should be conducive to long-term preservation of cardiac structure and function, which are aspects that remain to be established for GILZ. Thus, the demonstration of cardioprotective effects of GILZ delivery (via GILZ-overexpressing mesenchymal stem cells) along with its renoprotective effects (via use of the cell-permeable GILZ fusion protein as described above) raise the prospect of exogenous GILZ delivery as a novel cardiorenal-protective modality. This is an important consideration since cardiac injury/dysfunction adversely affects the kidney and kidney injury/dysfunction also adversely impacts the heart (i.e., cardiorenal syndromes; Zannad and Rossignol 2018).

In summary, important roles for GILZ in the cardiovascular system have been proposed in conditions associated with cardiac and vascular inflammation (Table 1). Nonetheless, major gaps remain in our understanding of the role of GILZ in various cardiovascular pathologies and the impact of longterm GILZ therapy on them.

\section{Conclusions}

Despite its discovery over 20 years ago, the role of GILZ in renal and cardiovascular physiologies remains largely unexplored. Nonetheless, and in light of the rather limited available information, it is apparent that GILZ regulates important physiologic functions in these organ systems and that abnormalities of GILZ can contribute to pathologies of the kidney and the cardiovascular system (Table 1). Thus, better understanding of the role of endogenous GILZ in regulating physiologic functions of the kidney and the cardiovascular system should lay the scientific foundation and rationale for GILZ therapy to alleviate the burden of renal and cardiovascular diseases.

Perspective. Since its discovery in 1997, a flurry of studies has established important roles for endogenous GILZ in regulation of innate and adaptive immune cells and their functional correlates. Thus, it is now clear that GILZ exerts cell- and context-specific immune regulatory/suppressive effects, thereby curbing inflammatory responses in a variety of conditions, including those affecting the cardiovascular system and the kidney (Fig. 1; Table 1). Importantly, the advent of GILZ-based options (e.g., cell-permeable GILZ fusion protein, synthetic GILZ peptide, GILZ-overexpressing mesenchymal stem cells) has raised the prospect of therapeutic GILZ delivery for a variety of conditions with underlying dysregulation of immune and inflammatory mechanisms, with the goal of avoiding deleterious effects associated with the use of glucocorticoids (Flynn et al., 2019; Vandewalle and Libert, 2020). The demonstrated effects of GILZ on immune and inflammatory responses take added clinical relevance and importance given emerging reports that glucocorticoids (e.g., DEXA) can be of benefit for those afflicted with the ongoing pandemic of COVID-19, likely because of their ability to curb its associated proinflammatory cytokine storm; the recommended dose of DEXA for this condition is $6 \mathrm{mg} /$ day (Wiersinga et al., 2020). Given the considerations described earlier, this dose is seemingly consistent with human equivalent dose of 1-6 mg for DEXA based on GILZ-related studies with mice (Cannarile et al., 2009; Vago et al., 2015; Nair and Jacob,
2016). Although COVID-19 is best known for causing pulmonary pathology, it is increasingly recognized that those afflicted with the disease are at increased risk of manifesting extrapulmonary multiorgan pathologies, including AKI; cardiac abnormalities, such as congestive heart failure; and endotheliitis (Gabarre et al., 2020; Guo et al., 2020a; Gupta et al., 2020; South et al., 2020; Varga et al., 2020). Thus, aside from the potential usefulness of GILZ therapy for pathologies of the cardiovascular system and kidney associated with other conditions, it is likely that therapeutic GILZ can also be useful for those affected with COVID-19 because of 1) GILZ-induced downregulation of immune and inflammatory responses, thereby reducing proinflammatory cytokine storm in the early phase of the disease process, and 2) GILZ-induced cytoprotection, which may attenuate/prevent long-term adverse outcomes. Nonetheless, it should be pointed out that our knowledge regarding therapeutic value of GILZ-based options comes from preclinical studies, and to date, no clinical trial has examined safety and efficacy of such therapies. Thus, the reported effects of GILZ, some of which were described in this report, should stimulate further research with the ultimate objective of harnessing its clinical usefulness for relevant human pathologies, including those affecting the kidney and the cardiovascular system.

\section{Authorship Contributions}

Performed data analysis: Mozaffari.

Wrote or contributed to the writing of the manuscript: Mozaffari.

\section{References}

Aguilar D, Strom J, and Chen QM (2014) Glucocorticoid induced leucine zipper inhibits apoptosis of cardiomyocytes by doxorubicin. Toxicol Appl Pharmacol 276: 55-62.

Aguilar DC, Strom J, Xu B, Kappeler K, and Chen QM (2013) Expression of glucocorticoid-induced leucine zipper (GILZ) in cardiomyocytes. Cardiovasc Toxicol 13:91-99.

Asselin-Labat ML, David M, Biola-Vidamment A, Lecoeuche D, Zennaro MC, Bertoglio J, and Pallardy M (2004) GILZ, a new target for the transcription factor FoxO3, protects $\mathrm{T}$ lymphocytes from interleukin-2 withdrawal-induced apoptosis. Blood 104:215-223.

Ayroldi E, Migliorati G, Bruscoli S, Marchetti C, Zollo O, Cannarile L, D'Adamio F, and Riccardi C (2001) Modulation of T-cell activation by the glucocorticoid-induced leucine zipper factor via inhibition of nuclear factor kappaB. Blood 98:743-753.

Ayyar VS, Almon RR, Jusko WJ, and DuBois DC (2015) Quantitative tissue-specific dynamics of in vivo GILZ mRNA expression and regulation by endogenous and exogenous glucocorticoids. Physiol Rep 3:e12382.

Ayyar VS, DuBois DC, Almon RR, and Jusko WJ (2017) Mechanistic multi-tissue modeling of glucocorticoid-induced leucine zipper regulation: integrating circadian gene expression with receptor-mediated corticosteroid pharmacodynamics. J Pharmacol Exp Ther 363:45-57.

Ayyar VS, DuBois DC, Almon RR, and Jusko WJ (2019) Modeling corticosteroid pharmacokinetics and pharmacodynamics, Part III: Estrous cycle and estrogen receptor-dependent antagonism of glucocorticoid-induced leucine zipper (GILZ) enhancement by corticosteroids. J Pharmacol Exp Ther 370:337-349.

Baban B, Khodadadi H, Vaibhav K, Marchetti C, Riccardi C, and Mozaffari MS (2020) Regulation of innate lymphoid cells in acute kidney injury: crosstalk between cannabidiol and GILZ. J Immunol Res 2020:6056373.

Baban B, Marchetti C, Khodadadi H, Malik A, Emami G, Lin PC, Arbab AS, Riccardi C, and Mozaffari MS (2018) Glucocorticoid-induced leucine zipper promotes neutrophil and T-cell polarization with protective effects in acute kidney injury. $J$ Pharmacol Exp Ther 367:483-493.

Baban B, Yin L, Qin X, Liu JY, Shi X, and Mozaffari MS (2017) The role of GILZ in modulation of adaptive immunity in a murine model of myocardial infarction. Exp Mol Pathol 102:408-414.

Ballegeer M, Vandewalle J, Eggermont M, Van Isterdael G, Dejager L, De Bus L, Decruyenaere J, Vandenbroucke RE, and Libert C (2019) Overexpression of gilz protects mice against lethal septic peritonitis. Shock 52:208-214.

Bereshchenko O, Migliorati G, Bruscoli S, and Riccardi C (2019) Glucocorticoidinduced leucine zipper: a novel anti-inflammatory molecule. Front Pharmacol 10: 308.

Bhalla V, Soundararajan R, Pao AC, Li H, and Pearce D (2006) Disinhibitory pathways for control of sodium transport: regulation of ENaC by SGK1 and GILZ. Am J Physiol Renal Physiol 291:F714-F721.

Bruscoli S, Donato V, Velardi E, Di Sante M, Migliorati G, Donato R, and Riccardi C (2010) Glucocorticoid-induced leucine zipper (GILZ) and long GILZ inhibit myogenic differentiation and mediate anti-myogenic effects of glucocorticoids. $J$ Biol Chem 285:10385-10396. 
Calmette J, Ellouze M, Tran T, Karaki S, Ronin E, Capel F, Pallardy M, Bachelerie F, Krzysiek R, Emilie D, et al. (2014) Glucocorticoid-induced leucine zipper enhanced expression in dendritic cells is sufficient to drive regulatory $\mathrm{T}$ cells expansion in vivo. J Immunol 193:5863-5872.

Cannarile L, Cuzzocrea S, Santucci L, Agostini M, Mazzon E, Esposito E, Muià C, Coppo M, Di Paola R, and Riccardi C (2009) Glucocorticoid-induced leucine zipper is protective in Th1-mediated models of colitis. Gastroenterology 136:530-541.

Cannarile L, Delfino DV, Adorisio S, Riccardi C, and Ayroldi E (2019) Implicating the role of GILZ in glucocorticoid modulation of T-cell activation. Front Immunol 10 1823.

Cannarile L, Zollo O, D’Adamio F, Ayroldi E, Marchetti C, Tabilio A, Bruscoli S, and Riccardi C (2001) Cloning, chromosomal assignment and tissue distribution of human GILZ, a glucocorticoid hormone-induced gene. Cell Death Differ 8:201-203.

Cari L, Ricci E, Gentili M, Petrillo MG, Ayroldi E, Ronchetti S, Nocentini G, and Riccardi C (2015) A focused real time PCR strategy to determine GILZ expression in mouse tissues. Results Immunol 5:37-42.

Cheng Q, Fan H, Ngo D, Beaulieu E, Leung P, Lo CY, Burgess R, van der Zwan YG, White SJ, Khachigian LM, et al. (2013) GILZ overexpression inhibits endothelial cell adhesive function through regulation of NF-кB and MAPK activity. J Immunol 191:424-433.

Cohen N, Mouly E, Hamdi H, Maillot MC, Pallardy M, Godot V, Capel F, Balian A Naveau S, Galanaud P, et al. (2006) GILZ expression in human dendritic cells redirects their maturation and prevents antigen-specific $\mathrm{T}$ lymphocyte response Blood 107:2037-2044.

Crome SQ and Ohashi PS (2018) Immunoregulatory functions of innate lymphoid cells. J Immunother Cancer 6:121.

D'Adamio F, Zollo O, Moraca R, Ayroldi E, Bruscoli S, Bartoli A, Cannarile L, Migliorati G, and Riccardi C (1997) A new dexamethasone-induced gene of the leucine zipper family protects T lymphocytes from TCR/CD3-activated cell death. Immunity 7:803-812.

Delfino DV, Agostini M, Spinicelli S, Vito P, and Riccardi C (2004) Decrease of Bcl-xL and augmentation of thymocyte apoptosis in GILZ overexpressing transgenic mice. Blood 104:4134-4141.

Dumeige L, Nehlich M, Viengchareun S, Perrot J, Pussard E, Lombès M, and Martinerie L (2020) Preterm birth is associated with epigenetic programming of transgenerational hypertension in mice. Exp Mol Med 52:152-165.

Dumeige L, Storey C, Decourtye L, Nehlich M, Lhadj C, Viengchareun S, Kappeler L, Lombès M, and Martinerie L (2017) Sex-specificity of mineralocorticoid target gene expression during renal development, and long-term consequences. Int $J \mathrm{Mol} S \mathrm{Sc}$ 18:457.

Ellouze M, Vigouroux L, Tcherakian C, Woerther PL, Guguin A, Robert O, Surenaud M, Tran T, Calmette J, Barbin T, et al. (2020) Overexpression of GILZ in macrophages limits systemic inflammation while increasing bacterial clearance in sepsis in mice. Eur J Immunol 50:589-602.

Esposito E, Bruscoli S, Mazzon E, Paterniti I, Coppo M, Velardi E, Cuzzocrea S, and Riccardi C (2012) Glucocorticoid-induced leucine zipper (GILZ) over-expression in T lymphocytes inhibits inflammation and tissue damage in spinal cord injury. Neurotherapeutics 9:210-225.

Fiol DF, Mak SK, and Kültz D (2007) Specific TSC22 domain transcripts are hypertonically induced and alternatively spliced to protect mouse kidney cells during osmotic stress. FEBS $J$ 274:109-124.

Flynn JK, Dankers W, and Morand EF (2019) Could GILZ be the answer to glucocorticoid toxicity in lupus? Front Immunol 10:1684.

Fridlender ZG, Sun J, Kim S, Kapoor V, Cheng G, Ling L, Worthen GS, and Albelda SM (2009) Polarization of tumor-associated neutrophil phenotype by TGF-beta: "N1" versus "N2" TAN. Cancer Cell 16:183-194.

Gabarre P, Dumas G, Dupont T, Darmon M, Azoulay E, and Zafrani L (2020) Acute kidney injury in critically ill patients with COVID-19. Intensive Care Med 46: $1339-1348$

Gu R, Lei B, Jiang C, and Xu G (2017) Glucocorticoid-induced leucine zipper suppresses ICAM-1 and MCP-1 expression by dephosphorylation of NF-кB p65 in retinal endothelial cells. Invest Ophthalmol Vis Sci 58:631-641.

Guo T, Fan Y, Chen M, Wu X, Zhang L, He T, Wang H, Wan J, Wang X, and Lu Z (2020a) Cardiovascular implications of fatal outcomes of patients with coronavirus disease 2019 (COVID-19). JAMA Cardiol 5:811-818.

Guo Y, Gu R, Yu J, Lei B, Gan D, and Xu G (2020b) Synthetic glucocorticoid-induced leucine zipper peptide inhibits lipopolysaccharide-induced ocular inflammation in rats. Ophthalmic Res 63:434-442.

Gupta A, Madhavan MV, Sehgal K, Nair N, Mahajan S, Sehrawat TS, Bikdeli B, Ahluwalia N, Ausiello JC, Wan EY, et al. (2020) Extrapulmonary manifestations of COVID-19. Nat Med 26:1017-1032.

Hahn RT, Hoppstädter J, Hirschfelder K, Hachenthal N, Diesel B, Kessler SM, Huwer H, and Kiemer AK (2014) Downregulation of the glucocorticoid-induced leucine zipper (GILZ) promotes vascular inflammation. Atherosclerosis 234 391-400.

Hoppstädter J, Diesel B, Eifler LK, Schmid T, Brüne B, and Kiemer AK (2012) Glucocorticoid-induced leucine zipper is downregulated in human alveolar macrophages upon Toll-like receptor activation. Eur J Immunol 42 1282-1293.

Hoppstädter J, Diesel B, Linnenberger R, Hachenthal N, Flamini S, Minet M, Leidinger P, Backes C, Grässer F, Meese E, et al. (2019) Amplified host defense by tolllike receptor-mediated downregulation of the glucocorticoid-induced leucine zipper (GILZ) in macrophages. Front Immunol 9:3111.
Hoppstädter J, Kessler SM, Bruscoli S, Huwer H, Riccardi C, and Kiemer AK (2015) Glucocorticoid-induced leucine zipper: a critical factor in macrophage endotoxin tolerance. J Immunol 194:6057-6067.

Hoppstädter J and Kiemer AK (2015) Glucocorticoid-induced leucine zipper (GILZ) in immuno suppression: master regulator or bystander? Oncotarget 6:38446-38457.

Jones SA, Perera DN, Fan H, Russ BE, Harris J, and Morand EF (2015) GILZ regulates Th17 responses and restrains IL-17-mediated skin inflammation. $J$ Autoimmun 61:73-80.

Ma Y, Yabluchanskiy A, Iyer RP, Cannon PL, Flynn ER, Jung M, Henry J, Cates CA, Deleon-Pennell KY, and Lindsey ML (2016) Temporal neutrophil polarization following myocardial infarction. Cardiovasc Res 110:51-61.

McCormick SM, Eskin SG, McIntire LV, Teng CL, Lu CM, Russell CG, and Chittur KK (2001) DNA microarray reveals changes in gene expression of shear stressed human umbilical vein endothelial cells. Proc Natl Acad Sci USA 98:8955-8960.

Muller OG, Parnova RG, Centeno G, Rossier BC, Firsov D, and Horisberger JD (2003) Mineralocorticoid effects in the kidney: correlation between alphaENaC, GILZ, and Sgk-1 mRNA expression and urinary excretion of $\mathrm{Na}+$ and $\mathrm{K}+$. J Am Soc Nephrol 14:1107-1115.

Nair AB and Jacob S (2016) A simple practice guide for dose conversion between animals and human. $J$ Basic Clin Pharm 7:27-31.

O'Byrne PM and Mejza F (2018) Advances in the treatment of mild asthma: recent evidence. Pol Arch Intern Med 128:545-549.

Qin X, Liu JY, Abdelsayed R, Shi X, Yu JC, Mozaffari MS, and Baban B (2016) The status of glucocorticoid-induced leucine zipper protein in the salivary glands in Sjögren's syndrome: predictive and prognostic potentials. EPMA J 7:3.

Rashmi P, Colussi G, Ng M, Wu X, Kidwai A, and Pearce D (2017) Glucocorticoidinduced leucine zipper protein regulates sodium and potassium balance in the distal nephron. Kidney Int 91:1159-1177.

Robert-Nicoud M, Flahaut M, Elalouf JM, Nicod M, Salinas M, Bens M, Doucet A, Wincker P, Artiguenave F, Horisberger JD, et al. (2001) Transcriptome of a mouse kidney cortical collecting duct cell line: effects of aldosterone and vasopressin. Proc Natl Acad Sci USA 98:2712-2716.

Ronco C, Bellomo R, and Kellum JA (2019) Acute kidney injury. Lancet 394: 1949-1964.

Soundararajan R, Zhang TT, Wang J, Vandewalle A, and Pearce D (2005) A novel role for glucocorticoid-induced leucine zipper protein in epithelial sodium channelmediated sodium transport. J Biol Chem 280:39970-39981.

South AM, Diz DI, and Chappell MC (2020) COVID-19, ACE2, and the cardiovascular consequences. Am J Physiol Heart Circ Physiol 318:H1084-H1090.

Suarez PE, Rodriguez EG, Soundararajan R, Mérillat AM, Stehle JC, Rotman S, Roger T, Voirol MJ, Wang J, Gross O, et al. (2012) The glucocorticoid-induced leucine zipper (gilz/Tsc22d3-2) gene locus plays a crucial role in male fertility. Mol Endocrinol 26:1000-1013.

Symowski C and Voehringer D (2017) Interactions between innate lymphoid cells and cells of the innate and adaptive immune system. Front Immunol 8:1422.

Ueda K, Fujiki K, Shirahige K, Gomez-Sanchez CE, Fujita T, Nangaku M, and Nagase M (2014) Genome-wide analysis of murine renal distal convoluted tubular cells for the target genes of mineralocorticoid receptor. Biochem Biophys Res Commun 445:132-137.

Vago JP, Galvão I, Negreiros-Lima GL, Teixeira LCR, Lima KM, Sugimoto MA Moreira IZ, Jones SA, Lang T, Riccardi C, et al. (2020) Glucocorticoid-induced leucine zipper modulates macrophage polarization and apoptotic cell clearance. Pharmacol Res 158:104842.

Vago JP, Tavares LP, Garcia CC, Lima KM, Perucci LO, Vieira EL, Nogueira CRC, Soriani FM, Martins JO, Silva PMR, et al. (2015) The role and effects of glucocorticoid-induced leucine zipper in the context of inflammation resolution. Immunol 194:4940-4950.

Valbuena Perez JV, Linnenberger R, Dembek A, Bruscoli S, Riccardi C, Schulz MH, Meyer MR, Kiemer AK, and Hoppstädter J (2020) Altered glucocorticoid metabolism represents a feature of macroph-aging. Aging Cell 19:e13156.

Vandewalle J and Libert C (2020) GILZ in sepsis: "poor is the pupil who does not surpass his master". Eur J Immunol 50:490-493.

Varga Z, Flammer AJ, Steiger P, Haberecker M, Andermatt R, Zinkernagel AS, Mehra MR, Schuepbach RA, Ruschitzka F, and Moch H (2020) Endothelial cell infection and endotheliitis in COVID-19. Lancet 395:1417-1418.

Vétillard M and Schlecht-Louf G (2018) Glucocorticoid-induced leucine zipper: finetuning of dendritic cells function. Front Immunol 9:1232.

Wiersinga WJ, Rhodes A, Cheng AC, Peacock SJ, and Prescott HC (2020) Pathophysiology, transmission, diagnosis, and treatment of coronavirus disease 2019 (COVID-19): a review. JAMA 324:782-793.

Yurtsever T, Streit F, Foo JC, Trifonova S, Kumsta R, Muller CP, Turner JD, Meyer $\mathrm{J}$, and Schote $\mathrm{AB}$ (2019) Temporal dynamics of cortisol-associated changes in mRNA expression of glucocorticoid responsive genes FKBP5, GILZ, SDPR, PER1, PER2 and PER3 in healthy humans. Psychoneuroendocrinology 102:63-67.

Zannad F and Rossignol P (2018) Cardiorenal syndrome revisited. Circulation 138 929-944.

Address correspondence to: Dr. Mahmood S. Mozaffari, Department of Oral Biology and Diagnostic Sciences, CL-2134, The Dental College of Georgia, Augusta University, 1120 15th St., Augusta, GA 30912-1128. E-mail: mmozaffa@augusta.edu 